\title{
Insurance problems among inflammatory bowel disease patients: results of a Dutch population based study
}

\author{
M G V M Russel, B M Ryan, P C Dagnelie, M de Rooii, J Sijbrandii, A Feleus, \\ M Hesselink, J W Muris, R Stockbrugger
}

See end of article for authors' affiliations

Correspondence to: Dr B Ryan, Department of Gastroenterology, University of Maastricht PO Box 5800, 6202 AZ Maastricht, the

Netherlands;

bbryan@planet.nl

Accepted for publication 16 September 2002

\begin{abstract}
Background and aims: The majority of patients with inflammatory bowel disease (IBD) have a normal life expectancy and therefore should not be weighted when applying for life assurance. There is scant literature on this topic. In this study our aim was to document and compare the incidence of difficulties in application for life and medical insurance in a population based cohort of IBD patients and matched population controls.

Methods: A population based case control study of 1126 IBD patients and 1723 controls. Based on a detailed questionnaire, the frequency and type of difficulties encountered when applying for life and medical insurance in matched IBD and control populations were appraised.

Results: In comparison with controls, IBD patients had an 87 -fold increased risk of encountering difficulties when applying for life assurance (odds ratio (OR) 87 (95\% confidence interval (Cl) 31-246)), with a heavily weighted premium being the most common problem. Patients of high educational status, with continuous disease activity, and who smoked had the highest odds of encountering such problems. Medical insurance difficulties were fivefold more common in IBD patients compared with controls (OR $5.4(95 \% \mathrm{Cl} 2.3-13))$ although no specific disease or patient characteristics were identified as associated with such difficulties.

Conclusions: This is the first detailed case control study that has investigated insurance difficulties among IBD patients. Acquiring life and medical insurance constituted a major problem for IBD patients in this study. These results are likely to be more widely representative given that most insurance companies use international guidelines for risk assessment. In view of the recent advances in therapy and promising survival data on IBD patients, evidence based guidelines for risk assessment of IBD patients by insurance companies should be drawn up to prevent possible discriminatory practices.
\end{abstract}

nflammatory bowel disease (IBD), comprising ulcerative colitis (UC), Crohn's disease (CD), and indeterminate colitis (IC), is the second most common chronic inflammatory condition next to rheumatoid arthritis. IBD presents mainly in young adulthood and has a variable and unpredictable clinical course. The social and economic implications are considerable, both for society and for the affected individual. ${ }^{1-3}$ Young adulthood is the age at which life and medical insurances are usually sought, and thus a diagnosis of IBD is likely to have a major impact on these processes. The scant literature that does exist on this important topic suggests that IBD patients encounter difficulties when applying for life assurance cover but no case control studies exist.

Life assurance risk assessment (underwriting) is based in principle on assessment of an individual's mortality risk compared with the "standard risk" for life assurance. ${ }^{5}$ The standard risk is an estimate of the pooled mortality risk of all insurees and is lower than that of the general population, as high risk individuals may be excluded and also those who seek insurance are self selected, usually from a more affluent sector of society and thus have a better survival rate than the general population.

In the last decade, a consistent body of evidence from prospective population based studies has shown that individuals with both UC and CD have a similar or marginally reduced life expectancy compared with the normal population. ${ }^{6-13} \mathrm{~A}$ slightly higher risk of mortality was found in UC patients within the first year of diagnosis ${ }^{7}$ and in certain subgroups of CD patients. ${ }^{814}$ There is also evidence that the mortality risk is higher in CD compared with UC. ${ }^{6}{ }^{15}$ Based on these data, it can be concluded that the vast majority of IBD patients should not be treated differently from healthy controls when applying for life assurance.

Medical insurance on the other hand reflects an individual's risk of morbidity and ensuing risk of incurring medical expenses. For an individual patient with IBD, there is a dearth of early prognostic factors that can reliably predict the natural history of the disease. Published IBD healthcare cost analyses are sparse, but in several studies CD patients incurred significantly more costs than UC patients ${ }^{316}{ }^{17}$ and a mere $4 \%$ of patients accounted for $75 \%$ of the IBD related medical costs. ${ }^{16}{ }^{17}$ In CD, Markov model analysis showed that surgery led to more durable remission than medical therapy, suggesting that surgery may reduce the overall long term costs of this condition. ${ }^{18}$

The current study investigated the frequency and type of life assurance and medical insurance application problems in a large population based group of Dutch IBD patients and compared this with matched population controls. In addition, disease factors that could influence such application difficulties were then assessed.

\section{METHODS}

Ethics approval

This study received the approval of our institution's ethics committee.

Abbreviations: IBD, inflammatory bowel disease; $C D$, Crohn's disease UC, ulcerative colitis; IC, indeterminate colitis; $O R$, odds ratio. 


\section{Patients}

All participating patients were part of the South Limburg Epidemiological Study on Inflammatory Bowel Disease (IBD-ZL) registry, a population based ongoing epidemiological study of over 2000 incident and prevalent cases of IBD, diagnosed since January 1987 in South Limburg, the Southern most province of the Netherlands. ${ }^{19}{ }^{20}$ Diagnosis of UC, CD, or IC was made in all cases using strict Lennard-Jones' criteria. $^{21}$ For the present study, only patients aged between 16 and 65 years, diagnosed between January 1987 and December 1999, were approached, as individuals beyond these age limits were considered unlikely to be applying for life or medical insurance.

\section{Controls}

The population of South Limburg in January 2000 was 648 882. ${ }^{22}$ The Registration Network of Family Practices (Registratie Netwerk Huisartsen) is a representative dynamic sampling frame of the South Limburg population and at the time of the current study included 81149 subjects. ${ }^{23}$ Frequency level age and sex matched controls were randomly selected from this register. Controls had no history of IBD, were resident in the study area, and had given permission to their general practitioner to be approached to participate in such studies.

\section{Questionnaire}

Questionnaires were sent to both patients and controls between March and May 2001, and written reminders were remitted after three and six weeks. A total of 1126 questionnaires were sent to IBD patients and 1723 to controls due to a lower anticipated response rate in controls. The questionnaires requested detailed sociodemographic information regarding age, sex, marital status, level of education, and smoking history. Education was stratified into three levels: low, medium, and high. Details of applications for life and medical insurance were sought including: whether an application had been made (in controls this was since the beginning of 1987 and in IBD patients since the time of diagnosis ); age at which insurance was sought; in the case of IBD patients whether or not IBD was reported to the insurance company; whether difficulties were encountered during the application for insurance; and the specific type of ensuing difficulty. In the patient questionnaire, additional disease related details were included, specifically: disease duration; specific diagnosis (UD, CD, or IC, as made by the treating physician); disease activity (one episode, fluctuating relapsing remitting course, or continuous activity $)^{24}$; use of immunosuppressive therapy; surgery; and the presence or absence of a stoma.

\section{Statistical analyses}

Statistical analyses were performed using SPSS version 10.0 for Windows (SPSS Inc. Chicago, Illinois, USA). Descriptive statistics were used to characterise patients and controls. Medians are given with $25-75 \%$ percentiles. When age associations were investigated, groups were divided into those above and below the median for $\chi^{2}$ analysis and was a continuous variable in logistic regression. Odds ratios (OR) are given with $95 \%$ confidence limits (95\% CI). A p value of less than 0.05 was considered significant.

Differences between the patient and control populations were analysed using $\chi^{2}$ analysis and logistic regression analysis. In the logistic regression significance was determined using the Wald test. Interactions between variables were sought, but none was significant. The dependent variable (insurance difficulty) was adjusted for age at time of application for the insurance policy, sex, smoking history, and educational level. In secondary analyses, non- significant variables were sequentially omitted and the remaining variables were further assessed within the same model. Differences are expressed as OR.

Analysis of patient factors that may be associated with an increased risk of encountering insurance difficulties was done
Table 1 Patient and control characteristics

\begin{tabular}{|c|c|c|}
\hline & Controls & IBD patients \\
\hline Total (n (\%)) & 824 & 781 \\
\hline UC & - & 424 (54) \\
\hline$C D$ & - & $311 \quad(40)$ \\
\hline IC & - & 46 (6) \\
\hline \multicolumn{3}{|l|}{$\operatorname{Sex}(n(\%))$} \\
\hline Male & $427(52)$ & 372 (48) \\
\hline Female & 397 (48) & $409 \quad(52)$ \\
\hline \multicolumn{3}{|l|}{ Median age (y) (25-75\%) } \\
\hline Male & 48 (39-59) & $47 \quad(36-58)$ \\
\hline Female & $40(34-52)$ & 39 (22-51) \\
\hline \multicolumn{3}{|l|}{ Smoking (n (\%)) } \\
\hline Current & $214(26)$ & 202 (26) \\
\hline Stopped & 272 (33) & 319 (41) \\
\hline Never & 312 (38) & 238 (30) \\
\hline Unspecified & $26(3)$ & 22 (3) \\
\hline \multicolumn{3}{|l|}{ Educational status (n (\%)) } \\
\hline Low & $263(32)$ & $276 \quad(35)$ \\
\hline Medium & 349 (42) & $359(46)$ \\
\hline High & $212(26)$ & 146 (19) \\
\hline $\begin{array}{l}\text { Median disease duration (y) } \\
(25-75 \%)\end{array}$ & - & \\
\hline UC & - & $7.3(4.3-9.6)$ \\
\hline$C D$ & - & 7.3(4.7-9.7) \\
\hline IC & - & $6.5(3.9-9.9)$ \\
\hline
\end{tabular}

by $\chi^{2}$ analysis followed by logistic regression analysis. Independent variables included: age at time of application for insurance; sex; educational level; smoking history; specific diagnosis (CD or UC); duration of disease; disease activity; stoma; history of surgery; and medication use. Analyses excluded IC patients, as the numbers were small.

\section{RESULTS}

\section{Baseline characteristics and response rate}

Questionnaires were completed and returned by 781/1126 $(69 \%)$ patients and by $824 / 1723(48 \%)$ controls. Patient and control characteristics are presented in table 1 .

\section{Life assurance}

Patients versus controls

During the stipulated time period, 199 controls (from 1987 to 1999) and 91 patients (since the time of diagnosis between 1987 and 1999) applied for a life assurance policy. Sixty of 91 (66\%) IBD patients compared with 7/199 (4\%) controls had encountered significant difficulties during this process. The crude OR for problematic life assurance application in patients compared with controls was 53.6 (22.5-128) and the corrected OR following logistic regression analysis was 87 (31-246) (table 2). By logistic regression, smoking, educational status, and age at application for insurance were not associated with a significantly increased odds of encountering life assurance difficulties (table 2). A marked increase in the premium accounted for $52 / 60(87 \%)$ of the problems encountered among patients and for 3/7 (43\%) problems among controls, with diverse problems accounting for the remainder.

\section{Patient factors associated with difficulties}

Univariate followed by logistic regression analysis showed that the following factors were associated with significantly more problems during life assurance application (table 3): continuous disease activity (OR $5.6(1.1-29.8)$ for continuous disease compared with one episode); patients with high educational status (OR 6.95 (1.13-42.7) and OR 8.8 (2.4-32.3) for high compared with low and medium educational status, respectively); and current smoker (OR $6.2(1.7-22.5)$ ) at the time of application. Patients with CD had a non- significantly reduced risk of life assurance application problems compared with UC patients (OR $0.4(0.1-1.3)$ ). 
Table 2 Comparison of life assurance difficulties in patients and controls

\begin{tabular}{lc}
\hline & OR $(95 \% \mathrm{Cl})$ \\
\hline Patients $(n=91) v$ controls $(n=199)^{*}$ & $87(31-246)$ \\
Educational level & $1.7(0.4-6.8)$ \\
High $v$ low & $0.5(0.1-1.8)$ \\
Middle $v$ low & $2.0(0.9-4.8)$ \\
Smokers $v$ non-smokers & $1.0(0.9-1.03)$ \\
Age at application & \\
\hline
\end{tabular}

The values presented are the result of logistic regression analysis. *Patients were significantly more likely to encounter difficulties when applying for a life assurance policy.

$\mathrm{OR}$, odds ratio; $95 \% \mathrm{Cl}, 95 \%$ confidence interval.

Table 3 Analysis of potential determinants of life assurance difficulties in inflammatory bowel disease (IBD) patients

\begin{tabular}{ll}
\hline & OR $(95 \% \mathrm{CI})$ \\
\hline $\begin{array}{l}\text { Diagnosis (CD } v \text { UC) } \\
\text { Age at application }\end{array}$ & $0.4(0.1-1.3)$ \\
Disease activity* & $1.0(0.9-1.1)$ \\
$\quad$ Continuous $v 1$ period & $5.6(1.1-29.8)$ \\
Continuous $v$ fluctuating & $4.4(1.1-17.5)$ \\
Fluctuating $v 1$ period & $1.3(0.3-5.0)$ \\
Disease duration & $1.1(0.9-1.4)$ \\
History of surgery (yes $v$ no) & $3.7(0.9-14.6)$ \\
History of immunosuppressive use & $0.9(0.3-2.8)$ \\
Sex (male $v$ female) & $1.6(0.5-4.9)$ \\
Educational level* & \\
High $v$ medium & $8.7(2.3-32.3)$ \\
High $v$ low & $6.9(1.1-42.7)$ \\
Smoking* & $6.2(1.7-22.5)$ \\
\hline
\end{tabular}

All values presented are results of logistic regression analysis. *These factors were significantly associated with an increased risk of incurring life assurance application difficulties in IBD patients. $\mathrm{OR}$, odds ratio; $95 \% \mathrm{Cl}, 95 \%$ confidence interval.

\section{Medical health insurance}

Patients versus controls

A total of 386 controls and 84 patients had applied for a medical insurance policy. Fourteen of 85 (16\%) IBD patients compared with 13/386 (3\%) controls had encountered difficulties following application for medical insurance, giving a crude OR of 5.7 (2.6-12.6) in patients compared with controls. By logistic regression analysis, the only factor significantly associated with medical insurance application difficulties was whether the individual was a patient or control; the OR for patients was $5.4(2.3-13)$ (table 4). Raised policy premium and limited coverage accounted for $10 / 14(71 \%)$ of the problems encountered among patients and for 5/13 (38\%) problems among controls. One patient was refused medical insurance cover altogether.

Patient factors associated with difficulties

Patient and disease related factors that could play a potential role in encountering medical insurance difficulties were then assessed, including: age at the time of application for life assurance, sex, smoking history, specific diagnosis (UC or CD), educational status, disease duration, disease activity, surgical history, and medication use. Univariate followed by logistic regression analysis revealed that none of these factors had a significant effect on the risk of encountering problems when applying for medical insurance (table 5).

\section{DISCUSSION}

This large study shows that IBD patients in the Netherlands in a population based setting were almost 90 -fold more likely than matched population controls to encounter significant difficulties when applying for life assurance during the period
Table 4 Comparison of medical insurance difficulties in patients and controls

\begin{tabular}{ll}
\hline & OR $(95 \% \mathrm{CI})$ \\
\hline Patients $(n=84) v$ controls $(n=386)^{*}$ & $5.4(2.3-13.0)^{*}$ \\
Educational level & $0.7(0.2-2.9)$ \\
$\quad$ High $v$ low & $0.6(0.15-2.4)$ \\
Middle $v$ low & $0.8(0.3-1.9)$ \\
Smokers $v$ non-smokers & $1.0(0.9-1.1)$ \\
Age at application & \\
\hline
\end{tabular}

The values presented are the result of logistic regression analysis. *Patients were significantly more likely to encounter difficulties when applying for a medical insurance policy.

$\mathrm{OR}$, odds ratio; $95 \% \mathrm{Cl}, 95 \%$ confidence interval.

\begin{tabular}{ll}
$\begin{array}{l}\text { Table } 5 \\
\text { encountering medical insurance problems in }\end{array}$ \\
$\begin{array}{l}\text { encis of potential determinants of } \\
\text { inflammatory bowel disease (IBD) patients }\end{array}$ \\
\hline \multicolumn{2}{c}{ OR $(95 \% \mathrm{CI})$} \\
\hline Diagnosis (CD $v$ UC) & $2.3(0.3-15.7)$ \\
Age at application & $1.0(0.8-1.1)$ \\
Disease activity & $1.3(0.2-9.0)$ \\
Continuous $v$ l period & $2.2(0.4-10.9)$ \\
Continuous $v$ fluctuating & $1.2(0.2-1.5)$ \\
Disease duration & $0.4(0.5-3.0)$ \\
History of surgery (yes $v$ no) & $2.6(0.2-27.5)$ \\
History of immunosuppressive use & $1.4(0.3-6.4)$ \\
Sex (male $v$ female) & - \\
Educational level & \\
Smoking (yes $v$ no) & $0.5(0.1-2.0)$ \\
\hline
\end{tabular}

The values presented are the results of logistic regression analysis. No patient factors were significantly associated with encountering medical insurance difficulties.

*There was a trend towards higher risk in higher educated but due to cells containing zero, OR could not be calculated.

The $\mathrm{p}$ value of the likelihood ratio test was significant at 0.042 . $\mathrm{OR}$, odds ratio; $95 \% \mathrm{Cl}, 95 \%$ confidence interval.

1987-1999, the most common problem being marked premium weighting. Among IBD patients, we found that continuous disease activity, smoking, and higher educational status were associated with the highest risk of encountering life assurance difficulties. While smoking is well known to be associated with increased mortality for a number of reasons, the other two associations are more difficult to explain. Continuous disease activity in itself is associated with higher morbidity but not higher mortality in IBD patients. ${ }^{11}$ Higher educational level is not known to be associated with higher mortality in IBD patients and the association between this factor and increased risk of encountering life assurance difficulties may be explained by the fact that individuals of higher educational status are likely to apply for larger life assurance policies, possibly in relation to a larger house mortgage application.

In principle, risk assessment of a life insurance applicant is based on assessment of a individual's risk relative to the "standard risk". If risk assessment of IBD patients takes into account the survival data and prognostic factors emanating from the large epidemiological studies of the past decade, then one might expect our data to show an association between identified risk factors for higher mortality in IBD patients and encountering life assurance application difficulties. Importantly however, our study showed no association between such identified factors, such as disease duration or specific diagnosis. ${ }^{6-81525}$ Extensive small bowel disease is recognised to be a risk factor for higher mortality in CD patients ${ }^{8}$ but unfortunately assessment of this factor was not feasible in the current study as details of disease extent were not sought in the 
self reported questionnaires as it was felt that this information would not be reliable.

Medical insurance also proved problematic for IBD patients in this study; they had a 5.5-fold increased risk of encountering difficulties, with heavy premium weighting being the most common problem. IBD patients clearly have a higher risk of incurring medical costs than the normal population and thus this finding is not unexpected. ${ }^{16}{ }^{17}$ What is surprising however is that in this study there was no association between medical insurance difficulties and factors known to be associated with increased morbidity among IBD patients and thus with greater healthcare utilisation such as older age, disease activity, ${ }^{24}$ and specific IBD diagnosis (CD $v$ UC)..$^{16} 26$

During the study period, $12 \%$ (91/781) of patients compared with $24 \%$ (199/824) of controls applied for life assurance and $11 \%(84 / 781)$ of patients versus $47 \%(386 / 824)$ of controls applied for medical insurance. This apparent difference is due to the fact that these figures only include IBD patients who had applied for an insurance policy since diagnosis (for example an IBD patient who applied for insurance in 1993 and developed IBD in 1997 would not be included in this figure), whereas for controls they include all people who had applied for insurance during the period 1987-1999.

Our study has several strengths. Firstly, it is a population based study including a large number of cases and controls and thus is representative of the overall situation in the Netherlands and not merely of referred cases. Secondly, to our knowledge this is the first population based study to investigate in detail the frequency and type of problems encountered by IBD patients when applying for life assurance and medical insurance. A small survey of patients and insurance companies on experiences and attitudes regarding insurance has the only reported data on this topic. ${ }^{4}$ That survey showed that IBD patients encountered difficulties while companies claimed not to discriminate against IBD patients, ${ }^{4}$ but the impact of these findings was limited by the fact that the study was small, not case controlled, and thus did not, as we did, compare an IBD population to population controls.

Potential limitations of this study include the fact that the study was carried out in a Dutch setting and thus the results may not be representative of other countries. However, most of the insurance companies involved purport to use international guidelines for risk assessment (unpublished data from questionnaire to insurance companies) and thus the results are indeed likely to be more widely applicable.

Secondly, the time period during which insurance was sought differed for patients and controls. For controls, this extended from 1987 to 1999, and controls would be expected to have been equally likely to seek insurance during any given year. For patients on the other hand, the inclusion period was from the time of diagnosis between 1987 and 1999 to the end of 1999, as fewer patients would have been diagnosed in the early years of the inclusion period; relatively greater numbers who sought insurance would have done so later in the study period. It is possible that insurance practices changed over the decade studied and that this was reflected in the difference between the two groups. However, it is extremely unlikely that any altered practices by insurance companies would account for the magnitude of the differences found between patient and control groups.

Thirdly, the higher response rate in IBD patients compared with controls could be a source of bias in this study. The response rate in controls was similar to that quoted in other postal questionnaire based studies. ${ }^{27}$ However, it is likely that both IBD patients and controls who had encountered insurance difficulties would have been more likely to reply to the questionnaire. Indeed, it is entirely plausible that a feeling of personal relevance with the study may play a stronger role in spurring controls who had encountered insurance difficulties to reply to the questionnaire whereas the higher response rate among IBD patients in the first place is an indication that patients already feel personally invested in these sorts of studies. Thus while this discrepant response rate is unlikely to affect the overall results and could actually underestimate the difference between patients and controls, we cannot exclude the possibility of this being a possible source of bias.

In conclusion, this study shows that the vast majority of Dutch IBD patients encounter significant problems when applying for life assurance cover and to a lesser extent when applying for medical insurance. This practice does not seem to have been influenced by the very promising survival data on IBD patients emanating from excellent studies performed in the past two decades. Evidence based guidelines for risk assessment of IBD patients by insurance companies should be drawn up to prevent possible discriminatory practices.

\section{ACKNOWLEDGEMENT}

This work was supported by a grant from the Dutch "Maag Lever Darm Stichting" (MLDS), and by Ferring, the Netherlands. The findings were presented in abstract form at the Dutch Society of Gastroenterology Spring Meeting, 2001

\section{Authors' affiliations}

M G V M Russel, B M Ryan, M de Rooii, A Feleus, M Hesselink,

R Stockbrugger, Department of Gastroenterology, University Hospital Maastricht, Maastricht, the Netherlands

P C Dagnelie, Department of Epidemiology, University of Maastricht, Maastricht, the Netherlands

J Sijbrandii, Centre for Data and Information Management (MEMIC), University of Maastricht, Maastricht, the Netherlands

J W Muris, Department of General Practice, University of Maastricht, Maastricht, the Netherlands

\section{REFERENCES}

1 Farmer $\mathbf{R}$. The social toll of inflammatory bowel disease. Can J Gastroenterol 1994;8:433-7.

2 Sonnenberg A. Disability and need for rehabilitation among patients with inflammatory bowel disease. Digestion 1992;51:168-78.

3 Blomqvist P, Ekbom A. Inflammatory bowel diseases: health care and costs in Sweden in 1994. Scand J Gastroenterol 1997:32:1 134-9.

Moody GA Mayberry JF life insurance and inflammatory bowel disease: is there discrimination against patients? Int J Colorectal Dis 1996:11:276-8.

5 Travis SP. Review article: insurance risks for patients with ulcerative colitis or Crohn's disease. Aliment Pharmacol Ther 1997;1 1:51-9.

6 Ekbom A, Helmick CG, Zack M, et al. Survival and causes of death in patients with inflammatory bowel disease: a population-based study. Gastroenterology 1992:103:954-60.

7 Langholz E, Munkholm P, Davidsen M, et al. Colorectal cancer risk and mortality in patients with ulcerative colitis. Gastroenterology 1992;103:1444-5

8 Munkholm P, Langholz E, Davidsen M, et al. Intestinal cancer risk and mortality in patients with Crohn's disease. Gastroenterology 1993; 105:1716-23.

9 Munkholm P. Crohn's disease-occurrence, course and prognosis. An epidemiologic cohort-study. Dan Med Bull 1997;44:287-302.

10 Witte J, Shivananda S, Lennard-Jones JE, et al. Disease outcome in inflammatory bowel disease: mortality, morbidity and therapeutic management of a 796-person inception cohort in the European Collaborative Study on Inflammatory Bowel Disease (EC-IBD). Scand J Gastroenterol 2000:35:1272-7.

11 Farrokhyar F, Swarbrick ET, Irvine EJ. A critical review of epidemiological studies in inflammatory bowel disease. Scand J Gastroenterol 2001;36:2-15.

12 Loftus EV Jr, Silverstein MD, Sandborn WJ, et al. Crohn's disease in Olmsted County, Minnesota, 1940-1993: incidence, prevalence, and survival. Gastroenterology 1998;114:1161-8.

13 Loftus EV Jr, Silverstein MD, Sandborn WJ, et al. Ulcerative colitis in Olmsted County, Minnesota, 1940-1993: incidence, prevalence, and survival. Gut 2000;46:336-43

14 Jess T, Winther KV, Munkholm P, et al. Mortality and causes of death in Crohn's disease: follow-up of a population-based cohort in Copenhagen County, Denmark. Gastroenterology 2002;122:1808-14.

15 Persson PG Bernell O, Leijonmarck CE, et al. Survival and cause-specific mortality in inflammatory bowel disease: a population-based cohort study. Gastroenterology 1996;1 10:1339-45

6 Hay JW, Hay AR. Inflammatory bowel disease: costs-of-illness. J Clin Gastroenterol 1992;14:309-17.

17 Hay AR, Hay JW. Inflammatory bowel disease: medical cost algorithms. I Clin Gastroenterol 1992:14:318-27.

18 Silverstein MD, Loftus EV, Sandborn WJ, et al. Clinical course and costs of care for Crohn's disease: Markov model analysis of a population-based cohort. Gastroenterology 1999;117:49-57. 
19 Russel MG, Dorant E, Volovics A, et al. High incidence of inflammatory bowel disease in the Netherlands: results of a prospective study. The South Limburg IBD Study Group. Dis Colon Rectum 1998:41:33-40.

20 Russel MG, Dorant E, Brummer R, et al. Appendectomy and the risk of developing ulcerative colitis or Crohn's disease: results of a large case-control study. South Limburg Inflammatory Bowel Disease Study Group. Gastroenterology 1997;1 13:377-82.

21 Lennard-Jones J. Classification of inflammatory bowel disease. Scand J Gastroenterol 1989;24(suppl 170):2-6.

22 Central Bureau voor Statistisch. Statistisch Jaarboek. Heerlen: Voorburg, 2000

23 Metsemakers J, Hoppener P, Knottenus J, et al. Computerized health information in the Netherlands: a registration network of family practices. Br J Gen Pract 1992;42:102-6.
24 Langholz E, Munkholm P, Davidsen M, et al. Course of ulcerative colitis: analysis of changes in disease activity over years. Gastroenterology 1994; 107:3-11.

25 Farrokhyar F, Swarbrick ET, Grace RH, et al. Low mortality in ulcerative colitis and Crohn's disease in three regional centers in England. Am J Gastroenterol 2001;96:501-7.

26 Blomqvist P, Feltelius N, Lofberg R, et al. A 10-year survey of inflammatory bowel diseases-drug therapy, costs and adverse reactions. Aliment Pharmacol Ther 2001;15:475-81.

27 Eaker S, Bergstrom R, Bergstrom A, et al. Response rate to mailed epidemiologic questionnaires: a population-based randomized trial of variations in design and mailing routines. Am J Epidemiol 1998; 147:74-82
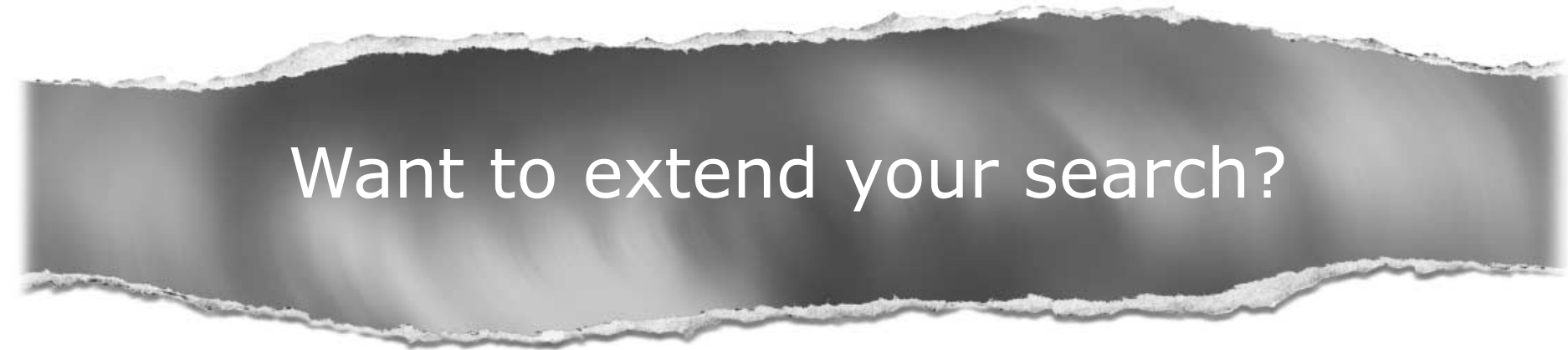

Cross journal searching

If you can't find what you are looking for in Gut you can extend your search across many of the more than 200 journals available for selection. You can restrict your search to specific subject areas (eg, clinical medicine, basic research), or select specific journals, or search all available titles.

\section{www.gutjnl.com}

